\title{
Do Mothers Have Worse Sleep Than Fathers? Sleep Imbalance, Parental Stress, and Relationship Satisfaction in Working Parents
}

\author{
Göran Härdelin (D) ${ }^{1,2}$ \\ Benjamin C Holding (iD) ${ }^{1,3}$ \\ Tim Reess ${ }^{2}$ \\ Anahita Geranmayeh ${ }^{1,2}$ \\ John Axelsson 1,2 \\ Tina Sundelin (iD) ${ }^{1,2}$ \\ 'Department of Clinical Neuroscience, \\ Karolinska Institutet, Stockholm, Sweden; \\ 2Department of Psychology, Stockholm \\ University, Stockholm, Sweden; \\ ${ }^{3}$ Department of Sociology, University of \\ Copenhagen, Copenhagen, Denmark
}

Correspondence: Tina Sundelin

Stress Research Institute, Department of Psychology, Stockholm, I06 9I, Sweden Email tina.sundelin@psychology.su.se
Purpose: Previous research indicates that mothers take a larger responsibility for child care during the night and that they have more disturbed sleep than fathers. The purpose of this study was to determine whether such a sleep imbalance exists in working parents of young children, and the extent to which it depends on the way sleep is measured. The study also examined whether imbalanced sleep between parents predicts parental stress and relationship satisfaction.

Methods: Sleep was measured for seven consecutive days in 60 parenting couples (average age of the youngest child: 3.3 years \pm SD 2.5 years). Actigraphs were worn across the week, and ratings of sleep, parental stress, and relationship satisfaction were made daily.

Results: Mothers perceived their sleep quality as worse $(b=-0.38$ scale units, $\mathbf{p}<0.001)$, with more wake periods $(b=+0.96$ awakenings, $\mathrm{p}<0.001)$ but with longer sleep duration $(b=$ +32.4 min, $\mathrm{p}<0.01)$ than fathers. Actigraphy data confirmed that mothers slept longer than fathers $(b=+28.03 \mathrm{~min}, \mathrm{p}<0.001)$, but no significant differences were found for wake time, number of awakenings or who woke up first during shared awakenings. Furthermore, there was no difference in whether mothers and fathers slept sufficiently. The level of sleep imbalance between parents did not predict parental stress. A larger imbalance in subjective sleep sufficiency predicted decreased relationship satisfaction for fathers $(b=-0.13$ scale units, $\mathrm{p}<0.01)$ but increased relationship satisfaction for mothers $(b=0.14$ scale units, $\mathrm{p}<0.05$ ). No other sleep imbalance measures predicted relationship satisfaction.

Conclusion: Our findings are in line with previous research on sleep in men and women in general, with longer sleep and subjective reports of sleep disturbances in women, rather than previous research on sleep in parents of young children. Thus, we found no evidence of a sleep imbalance when both parents have similar working responsibilities.

Keywords: parent, sleep, working, parental leave, equal, imbalance

\section{Introduction}

Parenthood is often characterized by sleep problems, such as poor sleep quality and shortened sleep duration. ${ }^{1-3}$ Previous studies indicate an imbalance in how each parent is affected by a child's birth, ${ }^{1,4}$ with mothers often reporting worse sleep than fathers. ${ }^{1,3,5,6}$ This is not surprising given that mothers are often the primary caregiver of children, ${ }^{7}$ and more likely to respond to their children's physical needs and worry more about the children during the night than fathers do. ${ }^{7}$ It is largely unknown how large this proposed sleep imbalance is, and whether it impacts the stress and well-being of family members. Given that a family is a dynamic system with reciprocal interactions among its members at all hours, 
and that disturbed sleep impacts subsequent daytime functioning for parents, ${ }^{8}$ including stress ${ }^{9-14}$ and relationship satisfaction, ${ }^{15,16}$ it seems likely that a sleep imbalance would also affect stress and relationship satisfaction. In the main bulk of previous research on sleep in couples, the role of primary caregiver is either unclear, ${ }^{3,5,6,17}$ or can be interpreted as being the mother's. ${ }^{4,7,14}$ In two of these studies, ${ }^{7,14}$ it was suggested that mothers also had the role of the primary caregiver at night, regardless of their parental leave status and regardless of whether both parents were working or not. However, there is a limited understanding of how parents objectively sleep when both are working, as most research in this field has neglected to assess parental leave status. ${ }^{1,3,5,6,17,18}$

Mothers generally report more sleep problems than fathers. ${ }^{1,3}$ For example, in a large longitudinal study, mothers reported a 62-minute reduction in nightly sleep duration during the first 3 months postpartum compared with pre-pregnancy, while fathers only reported a reduction of 13 minutes. ${ }^{1}$ Moreover, mothers continued to report worse sleep than fathers during the following six years. ${ }^{1}$ During a child's first time in life, one or both parents may be home from work, which could provide opportunities for more sleep. ${ }^{4}$ Indeed, there is some evidence that returning to work from parental leave results in self-reported sleep deprivation. ${ }^{19}$ However, one study found that self-reported night awakenings and duration of wakefulness do not differ between working and nonworking mothers of children 4-5 months old. ${ }^{14}$ A problem with these studies is that self-reports of sleep are often poorly correlated with objective measures, such as actigraphy and polysomnography, eg, ${ }^{20}$ and only a few studies have compared objectively measured sleep in couples with children. ${ }^{4,6,17}$ One of these focused on new parents and showed that mothers had less sleep at night than fathers one month postpartum, but that fathers obtained less total sleep when measured throughout the entire 24-h day. ${ }^{4}$ The other two studies showed that by the time the child is around 11 years old, there is no objectively measured difference in sleep duration, ${ }^{6,17}$ or sleep efficiency, ${ }^{17}$ between mothers and fathers, and even that mothers had less frequent long wake episodes compared to fathers. ${ }^{17}$ In all, there is evidence that women have more subjective sleep problems than men during their child's first 12 years, ${ }^{1,3}$ but although objectively measured sleep duration and sleep efficiency have not indicated such a difference, this has only been assessed in parents of children up to one month old or 11 years or older. There is thus limited knowledge about objective aspects of sleep in parents, particularly those with children between the ages of 1 and 11 years.

Another potential type of sleep disturbance in parents is who wakes up first due to a child's awakening. Evidence suggests that, for children ranging up to 18 years old, mothers adapt their sleep more to their children's sleep patterns than fathers do. ${ }^{5,6,17,18}$ For example, using actigraphy to assess sleep in triads of fathers, mothers, and children, one study found that children's sleep efficiency, long wake episodes, and wake-up minutes after sleep onset (WASO) were related to mothers' sleep efficiency, long wake episodes, and WASO the same night, while the fathers' sleep was not predicted by the children's sleep. ${ }^{17}$ Based on this, it seems likely that mothers are the first to wake up during the night due to a child awakening, but despite the existing evidence pointing in this direction, no previous study has actually assessed which parent typically wakes up first.

Mothers generally report higher levels of parental stress, the stress related to being a parent, ${ }^{21}$ when their child has sleep problems compared to when the child sleeps better. ${ }^{9,10,12}$ While there is less research on fathers, one study suggests that fathers are less affected by their child's sleep problems, reporting less depressive symptoms and a better family climate than mothers do. ${ }^{6}$ It has also been found that when fathers increase their general involvement in the child, parental stress for both parents is reduced..$^{13}$ Based on this, it is likely that a better balance in involvement, including a better balance in sleep and time spent caring for the child during the night, would lead to reduced stress in both parents.

More broadly, obtaining good sleep has been shown to be important for a range of social and romantic relationship aspects. ${ }^{22}$ For example, parents who sleep better are generally more satisfied with their relationship with their partner. ${ }^{15,23,24}$ Marital satisfaction has also been shown to be a predictor of perceived sleep quality in women postpartum. ${ }^{25}$ Perceived low support from the partner may reduce relationship satisfaction when experiencing parental stress, ${ }^{26}$ and stress in one partner has also been shown to impact relationship satisfaction negatively in both partners of a romantic couple. ${ }^{27}$ Therefore, it seems likely that poor sleep in one partner will lead to reduced relationship satisfaction in both partners through increased stress. However, the effects of sleep imbalance per se have not been discussed in the previous literature, and it is 
possible that an imbalance between parents may also impact satisfaction with the relationship.

In summary, a sleep imbalance between mothers and fathers of young children seems to be common, with the balance not restored until the children are considerably older. However, sleep has rarely been assessed objectively in both parents, and there is little knowledge of how working parents of young children sleep, and of who wakes up first at night because of the child's needs. Furthermore, any effects of differences/inequalities in sleep between the parents on parental stress and relationship satisfaction have not previously been studied. This study was designed to determine whether there is a subjective and/or objective sleep imbalance between working mothers and fathers with young children, and whether such an imbalance predicts parental stress and relationship satisfaction.

\section{Materials and Methods}

\section{Participants}

A sample of 146 parents ( 73 couples) agreed to participate in the study. The final sample was 60 couples, after exclusion and data loss (see below), with a mean age of 37.6 years $(\mathrm{SD}=6.8$ years). Each family had an average of 2 children $(\mathrm{SD}=1)$ and the age of the youngest child was on average 3.3 years old ( $\mathrm{SD}=2.5$ years). We advertised for parents of at least one child with disturbed sleep (mainly in digital meeting places set up to recruit potential volunteers, but also in social media groups for parents). Additional inclusion criteria were families with two parents where at least one of the parents claimed they woke up due to a child at least once per night for at least 4 nights a week, at least one child below the age of 12 , that both parents were willing to participate in the study, and that both parents worked at least $80 \%$ of full time. We included both married couples and cohabiting non-married couples but excluded couples where either parent had a sleep disorder not related to the child. We also excluded participants who reported commonly using substances that could interfere with sleep, and those with poor physical or mental health. Participants were not required to be the biological parents of the child. The target population was recruited from Stockholm County in Sweden, primarily through various social media websites and a web billboard for research subject recruitment. Each parent received a movie ticket for their participation. The study was approved by the Regional Ethics Committee in Stockholm (Dnr 2017/1742-31). The study was conducted in accordance with the declaration of Helsinki. Data was collected between 2017 and 2019.

\section{Procedure}

The study protocol was explained in written form to the participants. After receiving all necessary information, including potential risks, about the study, and before starting the data collection, participants signed an informed consent form. Following this, participants provided demographic information and baseline data.

They were instructed to wear actigraphs (MotionWatch 8, CamNtech, Cambridge, UK) on their non-dominant wrist for 7 consecutive nights and were clearly instructed that they could at any time discontinue their participation in the study. Participants were told to wear the actigraph continuously, except when taking a shower or a bath. Some participants did not wear the actigraph during work due to hygiene restrictions. Each morning they filled out a sleep diary and each night a questionnaire about their parental stress and relationship satisfaction that day.

\section{Measures}

\section{Sleep - Actigraphy}

Sleep scoring was performed based on the MotionWare algorithm with a sensitivity threshold $=20$. Actigraphy epoch duration for data acquisition was set to $30 \mathrm{sec}$ (because of a mistake, 7 participants had an epoch setting of $60 \mathrm{sec}$ ). Epoch duration within couples was consistent in all but one. Data from this couple were excluded for the analysis of shared awakenings (see below) but retained for all the remaining analyses. On average, parents had actigraphy data that could be used for analysis on 6.5 nights out of 7. Objective total sleep time (TST) was the sleep time in minutes between falling asleep and final wake up. WASO was the sum of the time in the actigraphy epochs scored as wake between falling asleep and final wake up. Sleep efficiency was computed as the TST divided by the total time in bed (TIB) between falling asleep and final wake up.

For shared awakenings within couples, we first applied thresholds with a minimum wake duration for awakenings ( $>1 \mathrm{~min}$ and $>5 \mathrm{~min}$, respectively) to every night of each participant separately. Only wake periods exceeding these thresholds were counted as wake periods. For each night within each couple, we then identified the time interval in which both partners were in bed simultaneously. Limited to this interval, we assessed how often wake epochs between partners overlapped in time. For each overlapping 
interval, we identified who woke up first (mother or father) or if both partners woke up simultaneously. Based on this procedure we derived the total number of shared awakenings and who woke up first during these shared awakenings, separately for both thresholds. All computations were performed using MATLAB (R2019a, MathWorks, Natick, MA, USA). Sleep sufficiency index (\%) was calculated by dividing the actigraphy TST with the stated habitual sleep need in the screening questionnaire and multiplying by 100 .

\section{Sleep - Sleep Diary}

Subjective sleep sufficiency was based on the question of how sufficient the previous night sleep was, on a scale ranging from 1 - "No, definitely too little" to 5 -"yes, definitely enough", from Karolinska Sleep Diary eg. ${ }^{28}$ Other subjective measures of sleep were number of times woken up during the night, sleep duration, and sleep quality on a scale ranging from 1 - "very low" to 5 "very high" sleep quality.

\section{Sleep Imbalance}

Measures of sleep imbalance were computed on a per night basis (see Supplementary Tables 1-3, for aggregated data across the entire week). Objective sleep imbalance for each measure was defined as the difference between mothers' and fathers' TST, WASO, sleep sufficiency index, number of wake periods longer than 1 minute, number of wake periods longer than 5 minutes, and who woke up first when having shared awakenings. Subjective sleep imbalance was measured as the difference between mothers' and fathers' perceived sleep quality, sleep sufficiency, number of awakenings during the night, and sleep duration.

\section{Parental Stress}

Daily variations in parental stress were measured with four questions from the Berry \& Jones Parental Stress Scale. ${ }^{21} \mathrm{We}$ included the questions that were most likely to change on a day-to-day basis, and the syntax of the questions were changed to concern the present day (Cronbach's alpha = 0.7). The questions were "Today, caring for my child(ren) took more time and energy than I had to give"; "Today, I worried whether I am doing enough for my child(ren)"; "Today I felt overwhelmed by the responsibility of being a parent"; "Today I felt strong affection for my child(ren)". Each one was scored between 1 - "strongly disagree" and 5 "strongly agree". The score of the last item was reversed and the average score formed the Parental Stress Index for that day.

\section{Relationship Satisfaction}

As an indicator of daily relationship satisfaction among partners, we used the item: "How satisfied are you with your interaction with your partner today?". Scores could range from 0 to 4 with 0 - "not satisfied at all" and 4 "very satisfied".

\section{Analyses}

Mixed-effect models were used to analyse whether a sleep imbalance between parents was observable in the data. Mixed-effect models were also used to assess the association between sleep imbalance and subsequent parental stress and relationship satisfaction. The models included couple-ID and participant-ID where appropriate as random effect factors to account for the non-independence of data. $\mathrm{R}$ (version 1.1.463) was used for the analysis. ${ }^{29} \mathrm{~A}$ p-value of $<0.05$ was considered significant. Data and analysis code can be found at https://osf.io/g9ck6/.

\section{Exclusions and Data Loss}

Out of the 73 couples who participated, thirteen were excluded from the analyses: one because only one partner filled out diary data, one because they were the same sex, 10 because actigraphy data was corrupt or missing for one or both partners, and one because the dates on the diaries were jumbled. Furthermore, the data from one couple was excluded for the shared awakenings analyses due to different epoch settings in their actigraphs.

\section{Results}

\section{Descriptive Statistics}

Median number of shared awakenings longer than 1 minute was 3 (Interquartile range (IQR) 2.4) per night and median number of shared awakenings longer than 5 minutes was 0.1 (IQR 0.3) per night. Of all nights registered for fathers, $41 \%$ had a TST of less than 6 hours, while for mothers this number was $21 \%$. All other descriptive statistics can be found in Table 1 .

\section{Sleep Imbalance}

\section{Subjective Measures}

Mothers reported more awakenings, worse sleep quality, and longer sleep duration than fathers did (see Table 2). There was, however, no difference between mothers' and fathers' subjective sleep sufficiency (see Table 2). 
Table I Descriptive Statistics for Sleep, Parental Stress, and Relationship Satisfaction. Both Parents in a Couple Wore Actigraphs and Filled Out Daily Ratings for One Week (Sleep Diary in the Morning and Ratings of Parental Stress and Relationship Satisfaction in the Evening)

\begin{tabular}{|c|c|c|c|}
\hline & Mothers $(n=67)$ & Fathers $(n=67)$ & Measure \\
\hline \multicolumn{4}{|l|}{ Objective measures } \\
\hline TST & $407(4 I)$ & $369(66)$ & Minutes, median (IQR) \\
\hline WASO & $59(30)$ & $58(33)$ & Minutes, median (IQR) \\
\hline Sleep efficiency & $85(6)$ & $83(6)$ & Percent, median (IQR) \\
\hline Number of wake periods longer than I minute & II (7) & II (7) & Count, median (IQR) \\
\hline Number of wake periods longer than 5 minutes & $I(I)$ & $\mathrm{I}(\mathrm{I})$ & Count, median (IQR) \\
\hline Sleep sufficiency index & $87(13)$ & $82(16)$ & Percent, median (IQR) \\
\hline \multicolumn{4}{|l|}{ Subjective measures } \\
\hline Sleep duration & $45 I(42)$ & $420(70)$ & Minutes, median (IQR) \\
\hline Number of awakenings & $2.6(1.1)$ & $1.6(1.0)$ & Count, mean (SD) \\
\hline Sleep quality & $2.9(0.6)$ & $3.3(0.6)$ & Scale I-5, mean (SD) \\
\hline Sleep sufficiency & $3(I . I)$ & $3.1(0.9)$ & Scale I-5, median (IQR) \\
\hline \multicolumn{4}{|l|}{ Parental stress } \\
\hline Parental stress index & $2.2(0.6)$ & $2.1(0.6)$ & Scale I-5, mean (SD) \\
\hline \multicolumn{4}{|l|}{ Relationship satisfaction } \\
\hline Relationship satisfaction & $2.5(0.8)$ & $2.6(0.6)$ & Scale I-5, mean (SD) \\
\hline
\end{tabular}

Notes: Objective sleep sufficiency was measured as the actual sleep divided by the time each participant assessed they would need for a sufficient night of sleep. Subjective sleep sufficiency was assessed by each participant every morning by on a scale from I - "no, definitely too little" and 5 - "yes, definitely enough". Parental Stress Index was measured with four questions, adapted from the Berry \& Jones Parental Stress Scale, ${ }^{21}$ which was scored between I - "strongly disagree" and 5 - "strongly agree". The score of the last item was reversed and the average score formed the Parental Stress Index for that day. Relationship satisfaction index was measured with one question, namely "How satisfied are you with your interaction with your partner today?, "which was scored by each parent every night between $0-4$ with 0 - "not satisfied at all" and 4 - "very satisfied".

Abbreviations: WASO, wake after sleep onset; TST, total sleep time; SE, standard error; CI, confidence interval IQR, interquartile range; SD, standard deviation.

\section{Objective Measures}

There was an imbalance in TST, where mothers' TST was longer than fathers' (See Table 2 and Figure 1). However, there was no imbalance in WASO, sleep efficiency, number of wake periods over 1 minute or number of wake periods over 5 minutes. There was also no difference in who woke up first during shared awakenings longer than 1 minute or longer than 5 minutes (see Table 2 and Figure 2). There was no difference between mothers' and fathers' sleep sufficiency index, ie actigraphy TST as a percentage of reported habitual sleep need (see Table 2).

\section{Parental Stress and Relationship Satisfaction}

The level of subjective or objective sleep imbalance between parents was not associated with parental stress, for neither mothers nor fathers (see Table 3). An increased imbalance in subjective sleep sufficiency predicted lower relationship satisfaction for fathers the following day, although this relationship was reversed for mothers (see
Table 4). No other subjective or objective sleep imbalance parameters predicted relationship satisfaction in either parent (see Table 4).

\section{Discussion}

The primary aim of this study was to determine whether there is a subjective and/or objective sleep imbalance between working mothers and fathers of young children. We found support for a subjective sleep imbalance in that mothers reported poorer sleep quality and having more waking periods, but with longer sleep duration, than fathers. However, only the sleep duration imbalance was confirmed by objective measures. Other objectively measured parameters showed no imbalance, neither in the number of wake periods nor WASO. Our finding that there is a sleep imbalance in subjective sleep quality and wake periods, unfavorable to the mother, is in line with previous research showing that mothers, and women in general, perceive their sleep as more disturbed than fathers/men do. ${ }^{1,3-5}$ The results also confirm previous 
Table 2 Sleep Imbalance Indicators (with Respect to How Mothers Sleep in Relation to Their Partner). Both Parents in a Couple Wore Actigraphs and Filled Out Daily Sleep Diary Ratings for One Week

\begin{tabular}{|c|c|c|c|c|c|c|c|}
\hline & \multirow[t]{2}{*}{ B } & \multirow[t]{2}{*}{ SE } & \multicolumn{2}{|c|}{$95 \% \mathrm{Cl}$} & \multirow[t]{2}{*}{ df } & \multirow[t]{2}{*}{ t-value } & \multirow[t]{2}{*}{ p-value } \\
\hline & & & Low & High & & & \\
\hline \multicolumn{8}{|l|}{ Objective measures } \\
\hline TST (min) & 31.39 & 7.32 & 17.0 & 45.9 & 59.23 & 4.29 & $<0.001$ \\
\hline WASO (min) & 2.55 & 3.48 & -4.32 & 9.41 & 58.59 & 0.73 & 0.47 \\
\hline Sleep efficiency (\%) & 1.00 & 0.86 & -0.69 & 2.70 & 58.64 & 1.16 & 0.25 \\
\hline Number of wake periods $>$ I minute & -0.35 & 0.69 & -1.71 & 1.01 & 57.49 & -0.51 & 0.61 \\
\hline Wake periods $>5$ minutes & -0.11 & 0.15 & -0.42 & 0.19 & 54.66 & -0.73 & 0.47 \\
\hline Waking up first in shared awakenings $>I$ minute & 3.67 & 4.78 & -0.42 & 0.19 & 52.36 & 0.77 & 0.45 \\
\hline Waking up first in shared awakenings $>5$ minutes & -15.89 & 12.75 & -41.0 & 9.75 & 34.19 & -1.25 & 0.22 \\
\hline Sleep sufficiency index & 3.61 & 2.16 & -0.65 & 7.88 & 51.65 & 1.67 & 0.10 \\
\hline \multicolumn{8}{|l|}{ Subjective measures } \\
\hline Sleep duration (min) & 33.5 & 9.1 & 15.5 & 51.4 & 46.44 & 3.69 & 0.001 \\
\hline Number of awakenings & 0.98 & 0.17 & 0.65 & 1.31 & 57.68 & 5.90 & $<0.001$ \\
\hline Sleep quality & -0.36 & 0.10 & -0.55 & -0.17 & 60.12 & -3.72 & $<0.001$ \\
\hline Sleep sufficiency & -0.24 & 0.12 & -0.47 & $<0.01$ & 58.90 & -2.07 & 0.04 \\
\hline
\end{tabular}

Notes: Negative number means the number is less for the mother. Sleep efficiency measured in percent. Number of wake measured in counts. Waking up first in shared awakenings was measured as the percentage of shared awakenings when the father or mother woke up first. Sleep sufficiency index measured in percent. Sleep duration measured in minutes. Subjective sleep quality and sleep sufficiency measured in scales $\mathrm{I}-5$.

Abbreviations: WASO, wake after sleep onset; TST, total sleep time; SE, standard error; Cl, confidence interval.

findings that women in general have longer subjective and objective sleep duration than men. ${ }^{30,31}$ Previous research has suggested that the difference in sleep duration between men and women may diminish directly after the birth of a new child and then be slowly restored as the child grows older, ${ }^{1}$ and return to normal levels once the child is around 11 years old. ${ }^{17}$ Our findings indicate that this happens earlier, once both parents are back to work after parental leave.

The second aim was to determine whether sleep imbalance predicts parental stress and relationship satisfaction. We found very little support for this, as sleep imbalance was not clearly related to parental stress or relationship satisfaction the following day, neither for mothers nor for fathers. The fact that we did not find such an association may be due to the lack of a consistent sleep imbalance in this group. A large imbalance in childcare involvement during the night may still lead to increased parental stress in both parents, but this has hardly been studied before. Although one study found that parental stress for both parents decreased when fathers increased their general involvement in the child, ${ }^{13}$ it remains unclear how the balance of night-specific tasks affects subjective and objective sleep, and impacts parents' stress levels.

Previous research has shown that mothers are more likely to adapt their sleep patterns to their children's sleep patterns and are more disturbed by children at night than fathers are, ${ }^{5,6,17,18}$ but to our knowledge, no study has previously measured who wakes up first during shared awakenings. As mothers' objectively measured sleep has been found to be associated with both children's and fathers' sleep, while fathers' sleep is associated with the mothers' but not the child's sleep, ${ }^{17}$ one would expect mothers to wake up first when needing to tend to the child. Surprisingly, we found no difference in who woke up first during shared awakenings, neither for wake periods longer than 1 minute nor wake periods longer than 5 minutes. Although this way of measuring who wakes up first in mutual awakenings is based on intervals of 30 seconds and we are not able to assess who wakes up first within each interval, it indicates that there is no tendency for mothers or fathers to consistently wake up before their partner during the night. A limitation is that we could not directly relate these shared awakenings to a child's awakenings, as we do not have data on the children's sleep. It is possible that future and more detailed analyses, also including awakenings of the child, will shed light on the dynamics of how child awakenings affect when parents wake up during the night, and which parent tends to wake up first.

The paradox of women sleeping longer while perceiving their sleep as more disturbed has been attributed to 


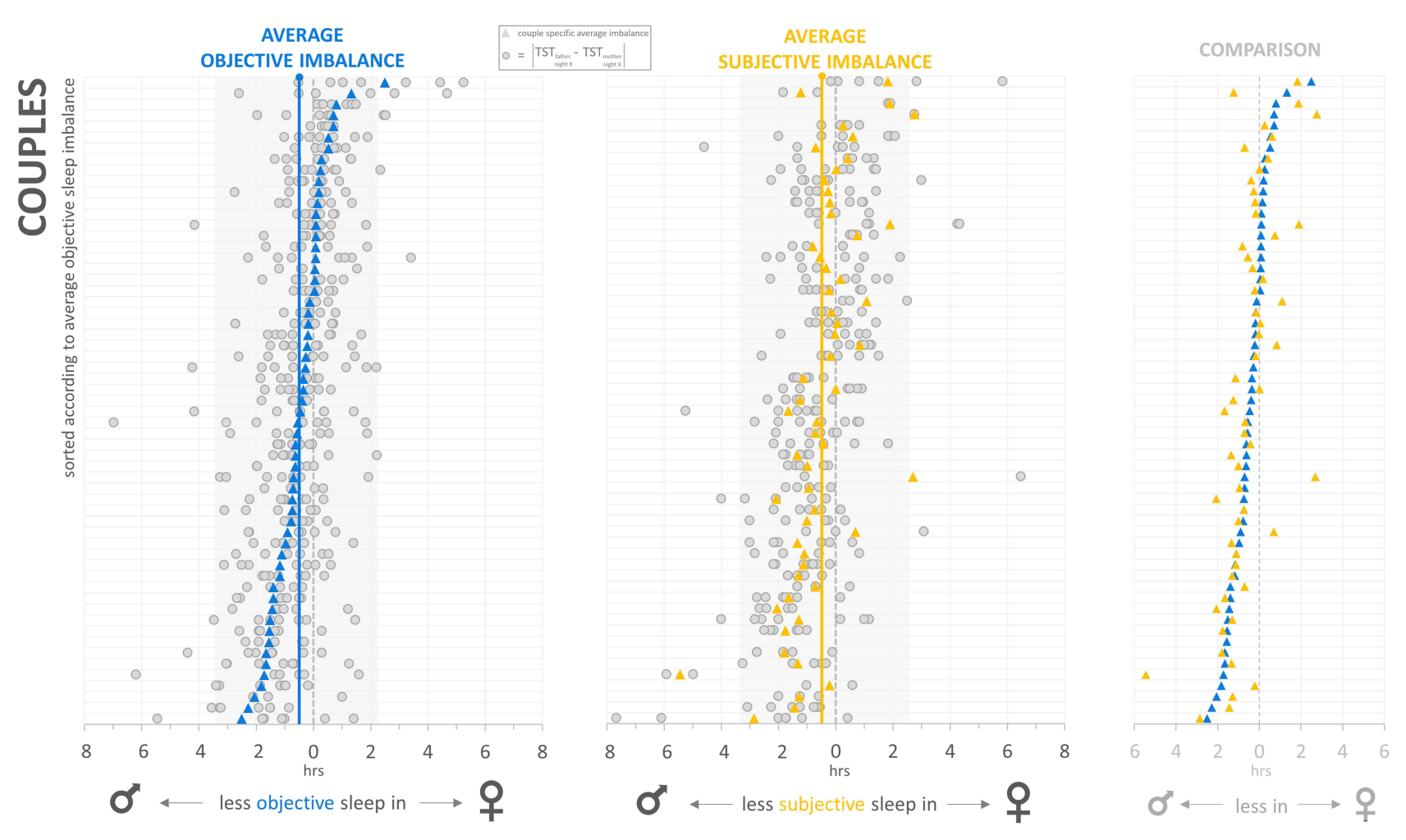

Figure I Sleep imbalance in objective and subjective sleep duration. Objective sleep duration is TST and subjective sleep duration is assessed by participants in daily sleep diaries. Rows indicate couples, dots represent imbalance in hours for each night within a given couple. Triangles represent couple-specific average imbalances. Solid vertical lines indicate the average sample specific imbalance measure. Dashed vertical lines provide a perfect balance reference. Shaded areas comprise $95 \%$ of raw data. Left panel: Objective sleep imbalance measurements based on actigraphy. Couples are sorted according to imbalance from high in fathers to high in mothers. Mothers have 28.0 min $(\mathrm{SE}=7 . \mathrm{I}, \mathrm{p}<0.00 \mathrm{I})$ longer objective sleep duration than men. Middle panel: Subjective sleep imbalance measurements based on questionnaire data. Couples are sorted as in the left panel. Mothers have 32.4 min $(\mathrm{SE}=9.3, \mathrm{p}=0.00 \mathrm{I})$ longer subjective sleep duration than men. Right panel: Comparison between objective and subjective imbalance. Blue triangles represent objective couple-specific average imbalance and yellow triangles represent subjective measurements. Note that for two couples, data was only available for objective measurements.

Abbreviations: TST, total sleep time; SE, standard error.

differences in sex hormones, genetics, and cultural and social demands, but is not fully understood. ${ }^{32,33}$ One explanation may be that while women objectively sleep longer, they have an even longer preferred sleep duration. ${ }^{31}$ Evidence of this comes, for example, from a questionnaire-based study of a large group of women and men, finding that women slept 14 minutes longer on workdays and 27 minutes longer on leisure days, but had 32 minutes longer preferred sleep duration than men. ${ }^{31}$ This is in line with our findings, showing that despite mothers' sleeping longer than fathers, both parents considered their sleep as equally sufficient.

One limitation with this study is that we did not measure the child's sleep. Even though a child's awakening is an external stressor that could wake up parents, there are of course many other potential reasons why parents wake up. Future studies would benefit from including measures of the child's sleep in order to have a better understanding of what triggered the mutual awakenings of the parents. It is possible that other factors that may have affected both sleep and the study outcomes, such as daily variations in health or drug abuse. Although we screened participants for general health and drug use, we did not measure these directly, and they are thus potential confounds. Afurther limitation is the relatively small sample size. Fortunately, each couple has an average of 6.5 days (out of 7) of measures and the analyses are all within-couple, which strengthens our conclusions. Although this is one of the largest studies looking at objective sleep in both parents with regard to sample size and number of nights, and the only study focusing on parents of children between 1 and 12 years old, along with the effects of sleep imbalance, future studies would benefit from including a larger and more diverse sample of parents. As the study sample is also rather homogeneous, future studies would benefit from including more participants (or stratified sampling) in order to have the power to draw conclusions about these relationships in different groups. When it comes to 


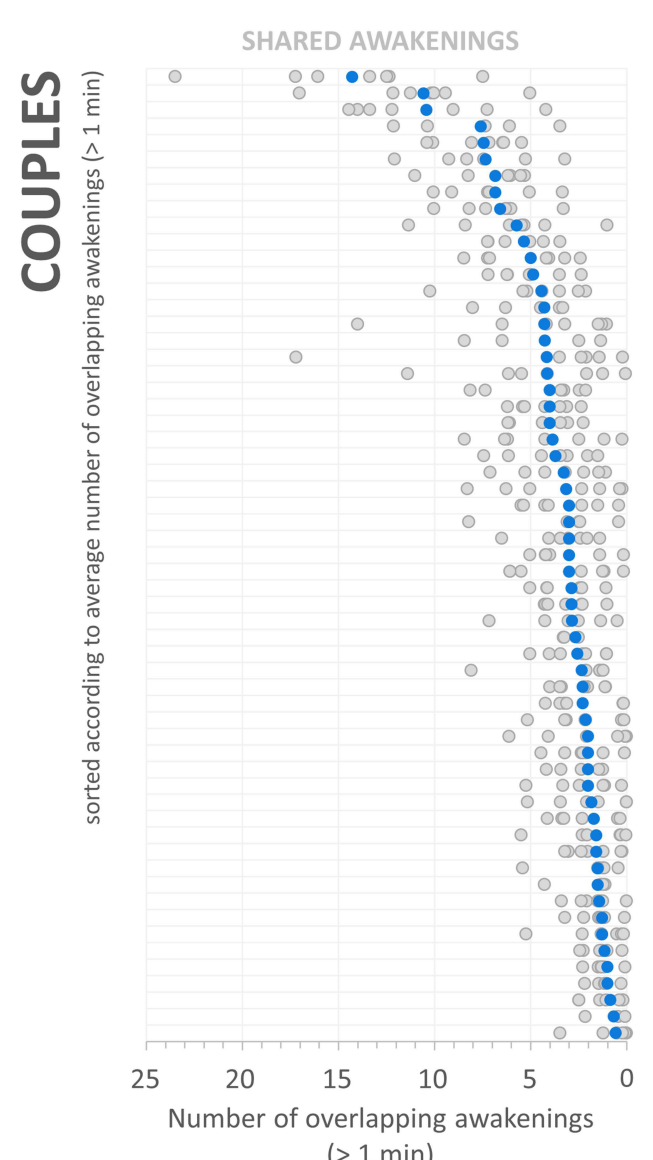

(> $1 \mathrm{~min}$ )

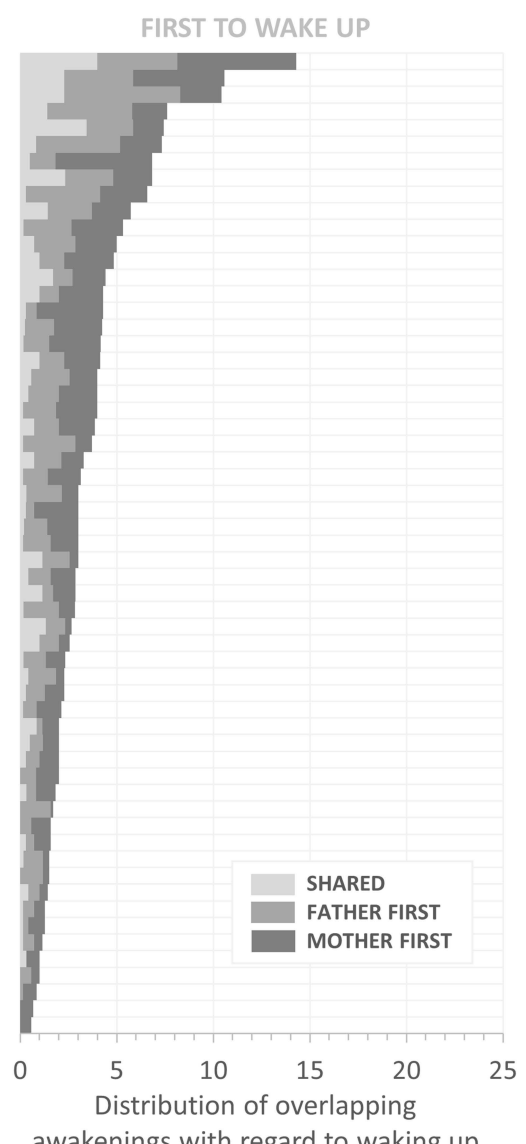

awakenings with regard to waking up

Figure 2 Characteristics of shared awakenings according to actigraphy in parents during the night. Objective sleep imbalance between fathers and mothers in who wakes up first. Rows indicate couples. Left panel: Gray dots represent the number of shared awakenings with duration longer than I min per night (note that data has been jittered in order to minimize overplotting of shared awakenings). Blue dots indicate the average number of shared awakenings within each couple based on objective actigraphy data. Data is sorted according to the couple-specific average number of shared awakenings from low to high. Median number of shared awakenings longer than I minute was 3 (IQR 2.4). Right panel: Stacked bars indicate the distribution of shared awakenings with respect to who woke up first; there was no significant difference between mothers and fathers $(b=3.58, S E=4.84, p=0.46)$.

Abbreviation: Min, minutes.

limitation in measures, we used a single question on relationship interaction satisfaction taken from the Relationship Interaction Satisfaction scale. ${ }^{34}$ Although this scale has been used previously to assess relationship satisfaction, there may be better ways to measure this aspect. One of the strengths of this study is the simultaneous use of objective measures of sleep in both parents, allowing for the assessment of within-couple sleep dynamics by looking at the epoch-by-epoch data. Considering that few studies have previously examined sleep in families where both parents work, it is a further strength that all participating parents were working at least $80 \%$ of full time. Although we did not find any large effects of imbalanced sleep in this study, the concept of sleep imbalance may be useful in other settings. For example, the effect of a sleep imbalance may be larger for parents on parental leave than for working parents, or in couples where sleep loss in general is bigger, such as for parents with younger children. It is also possible that larger sleep imbalances would be found in working parents from countries with less gender equality. ${ }^{35}$

\section{Conclusions}

This study finds that although there is an imbalance in subjective sleep quality, unfavorable to the mother, between working parents of young children, this was not observable in the corresponding actigraphy data. However, both the objective and subjective data on sleep length show that sleep length was longer in mothers. Overall, our findings are in line with previous research on sleep in men and women in general, rather than previous research on sleep in parents of young children. Our data indicate that when both parents are back to work, mothers do not show more objective sleep 
Table 3 Parental Stress Prediction by Sleep Imbalance Variables (How Differences in Sleep Between Mother and Father Predict Subsequent Daily Parental Stress Within Couples)

\begin{tabular}{|c|c|c|c|c|c|c|c|}
\hline & \multirow[t]{2}{*}{ b } & \multirow[t]{2}{*}{ SE } & \multicolumn{2}{|c|}{ 95\% Cl } & \multirow[t]{2}{*}{ df } & \multirow[t]{2}{*}{ t-value } & \multirow[t]{2}{*}{ p-value } \\
\hline & & & Low & High & & & \\
\hline \multicolumn{8}{|l|}{ Objective sleep measures } \\
\hline \multicolumn{8}{|l|}{ WASO imbalance model } \\
\hline Intercept & 2.04 & 0.09 & 1.87 & 2.21 & 168.60 & 23.50 & $<0.001$ \\
\hline WASO imbalance & 0.00 & 0.00 & -0.00 & 0.00 & 734.50 & 0.92 & 0.36 \\
\hline Sex & 0.14 & 0.11 & -0.07 & 0.35 & 100.40 & 1.29 & 0.20 \\
\hline WASO imbalance* sex & 0.00 & 0.00 & -0.00 & 0.00 & 665.50 & -0.31 & 0.76 \\
\hline \multicolumn{8}{|l|}{ Sleep sufficiency index imbalance model } \\
\hline Intercept & 2.08 & 0.09 & 1.89 & 2.26 & 151.80 & 21.93 & $<0.001$ \\
\hline Sleep sufficiency index imbalance & 0.00 & 0.00 & -0.01 & 0.01 & 655.80 & 0.09 & 0.93 \\
\hline Sex & 0.05 & 0.12 & -0.18 & 0.27 & 91.71 & 0.40 & 0.69 \\
\hline Sleep sufficiency index imbalance* sex & 0.00 & 0.00 & -0.00 & 0.01 & 592.70 & 1.06 & 0.29 \\
\hline \multicolumn{8}{|l|}{ Wake up periods $>$ I minute imbalance model } \\
\hline Intercept & 2.09 & 0.09 & 1.91 & 2.27 & 179.48 & 23.20 & $<0.001$ \\
\hline Wake up periods $>$ I minute imbalance & 0.00 & 0.01 & -0.02 & 0.01 & 722.92 & -0.54 & 0.59 \\
\hline Sex & 0.10 & 0.11 & -0.12 & 0.32 & 109.40 & 0.85 & 0.40 \\
\hline Wake up periods $>$ I minute imbalance* sex & 0.01 & 0.01 & -0.02 & 0.03 & 652.94 & 0.66 & 0.51 \\
\hline \multicolumn{8}{|l|}{ Wake up periods $>5$ minutes imbalance model } \\
\hline Intercept & 2.04 & 0.08 & 1.88 & 2.21 & 140.53 & 24.38 & $<0.001$ \\
\hline Wake up periods longer than 5 minutes imbalance & 0.02 & 0.03 & -0.03 & 0.07 & 720.81 & 0.69 & 0.49 \\
\hline Sex & 0.17 & 0.10 & -0.03 & 0.37 & 82.26 & 1.63 & 0.11 \\
\hline Wake up periods $>5$ minutes imbalance* sex & -0.02 & 0.03 & -0.09 & 0.05 & 666.03 & -0.66 & 0.51 \\
\hline \multicolumn{8}{|c|}{ Waking up first during shared wake periods $>$ I minute imbalance model } \\
\hline Intercept & 2.04 & 0.09 & 1.86 & 2.22 & 200.20 & 21.96 & $<0.001$ \\
\hline Waking up first during shared wake periods $>$ I minute imbalance & 0.00 & 0.00 & -0.00 & 0.00 & 608.60 & -0.33 & 0.74 \\
\hline Sex & 0.16 & 0.12 & -0.07 & 0.40 & 115.40 & 1.38 & 0.17 \\
\hline Waking up first during shared wake periods $>$ I minute imbalance* sex & 0.00 & 0.00 & -0.00 & 0.00 & 585.40 & -0.07 & 0.94 \\
\hline \multicolumn{8}{|l|}{ Subjective sleep measures } \\
\hline \multicolumn{8}{|l|}{ Sleep sufficiency imbalance model } \\
\hline Intercept & 2.09 & 0.09 & 1.92 & 2.26 & 169.28 & 24.12 & $<0.001$ \\
\hline Sleep sufficiency imbalance & -0.01 & 0.04 & -0.08 & 0.07 & 672.69 & -0.14 & 0.89 \\
\hline Sex & 0.07 & 0.11 & -0.14 & 0.28 & 102.45 & 0.67 & 0.50 \\
\hline Sleep sufficiency imbalance*sex & 0.04 & 0.05 & -0.07 & 0.14 & 665.25 & 0.70 & 0.48 \\
\hline \multicolumn{8}{|l|}{ Number of awakenings imbalance model } \\
\hline Intercept & 2.04 & 0.09 & 1.87 & 2.21 & 176.01 & 23.13 & $<0.001$ \\
\hline Number of awakenings imbalance & 0.03 & 0.03 & -0.02 & 0.08 & 697.44 & I.II & 0.27 \\
\hline Sex & 0.12 & 0.11 & -0.10 & 0.33 & 107.63 & 1.05 & 0.30 \\
\hline Number of awakenings imbalance* sex & 0.00 & 0.04 & -0.08 & 0.07 & 663.42 & -0.07 & 0.95 \\
\hline
\end{tabular}

Notes: Imbalance numbers are undirected, larger numbers mean more imbalance regardless of which parent had more or less. Parental Stress Index measured with four questions, adapted from the Berry \& Jones Parental Stress Scale, ${ }^{21}$ which was scored between I - "strongly disagree" and 5 - "strongly agree". The score of the last item was reversed and the average score formed the Parental Stress Index for that day. WASO and TST measured in minutes. Number of wake periods measured in numbers. Waking up first in shared awakenings was measured as the percentage of total shared awakenings. Sleep sufficiency index measured in percentage. Sleep duration measured in minutes. Subjective sleep sufficiency measured in scale I-5.

Abbreviations: Sex, the effect of being female; WASO, wake after sleep onset; TST, total sleep time; SE, standard error; CI, confidence interval. 
Table 4 Relationship Satisfaction Prediction by Sleep Imbalance Variables (How Differences in Sleep Between Mother and Father Predict Subsequent Daily Relationship Satisfaction Within Couples)

\begin{tabular}{|c|c|c|c|c|c|c|c|}
\hline & \multirow[t]{2}{*}{ b } & \multirow[t]{2}{*}{ SE } & \multicolumn{2}{|c|}{$95 \% \mathrm{Cl}$} & \multirow[t]{2}{*}{ df } & \multirow[t]{2}{*}{ t-value } & \multirow[t]{2}{*}{ p-value } \\
\hline & & & Low & High & & & \\
\hline \multicolumn{8}{|l|}{ Objective sleep measures } \\
\hline \multicolumn{8}{|l|}{ WASO imbalance model } \\
\hline Intercept & 2.57 & 0.10 & 2.36 & 2.77 & 166.40 & 24.53 & $<0.001$ \\
\hline WASO imbalance & 0.00 & 0.00 & -0.00 & 0.00 & 744.20 & 0.12 & 0.91 \\
\hline Sex & -0.05 & 0.12 & -0.29 & 0.19 & 108.10 & -0.40 & 0.69 \\
\hline WASO imbalance* sex & 0.00 & 0.00 & -0.01 & 0.01 & 581.70 & 0.02 & 0.98 \\
\hline \multicolumn{8}{|l|}{ Sleep sufficiency index imbalance model } \\
\hline Intercept & 2.60 & 0.11 & 2.37 & 2.82 & 153.20 & 22.86 & $<0.001$ \\
\hline Sleep sufficiency index imbalance & 0.00 & 0.00 & -0.01 & 0.01 & 656.60 & -0.21 & 0.83 \\
\hline Sex & 0.07 & 0.13 & -0.19 & 0.32 & 98.88 & 0.52 & 0.61 \\
\hline Sleep sufficiency index imbalance* sex & -0.01 & 0.00 & -0.02 & 0.00 & 509.30 & -1.56 & 0.12 \\
\hline \multicolumn{8}{|l|}{ Wake up periods $>$ I minute imbalance model } \\
\hline Intercept & 2.46 & 0.11 & 2.24 & 2.67 & 178.02 & 22.45 & $<0.001$ \\
\hline Wake up periods $>$ I minute imbalance & 0.02 & 0.01 & -0.00 & 0.05 & 731.81 & 1.93 & 0.054 \\
\hline Sex & 0.02 & 0.13 & -0.23 & 0.27 & 118.60 & 0.17 & 0.87 \\
\hline Wake up periods $>$ I minute imbalance* sex & -0.02 & 0.02 & -0.05 & 0.02 & 569.25 & -0.95 & 0.35 \\
\hline \multicolumn{8}{|l|}{ Wake up periods $>5$ minutes imbalance model } \\
\hline Intercept & 2.55 & 0.10 & 2.36 & 2.75 & $|37.4|$ & 25.48 & $<0.001$ \\
\hline Wake up periods $>5$ minutes imbalance & 0.02 & 0.03 & -0.05 & 0.08 & 729.57 & 0.46 & 0.65 \\
\hline Sex & 0.02 & 0.11 & -0.20 & 0.24 & 87.52 & 0.18 & 0.86 \\
\hline Wake up periods $>5$ minutes imbalance* sex & -0.06 & 0.05 & -0.15 & 0.03 & 617.26 & -1.31 & 0.19 \\
\hline \multicolumn{8}{|c|}{ Waking up first during shared wake periods $>$ I minute imbalance model } \\
\hline Intercept & 2.62 & 0.12 & 2.39 & 2.85 & 196.10 & 22.40 & $<0.001$ \\
\hline Waking up first during shared wake periods $>$ I minute imbalance & 0.00 & 0.00 & -0.00 & 0.00 & 630.70 & -0.63 & 0.53 \\
\hline Sex & -0.16 & 0.14 & -0.43 & 0.12 & 129.50 & -1.12 & 0.26 \\
\hline Waking up first during shared wake periods $>$ I minute* sex & 0.00 & 0.00 & -0.00 & 0.01 & 568.40 & 1.05 & 0.30 \\
\hline \multicolumn{8}{|l|}{ Subjective sleep measures } \\
\hline \multicolumn{8}{|l|}{ Sleep sufficiency imbalance model } \\
\hline Intercept & 2.71 & 0.10 & 2.50 & 2.91 & 164.74 & 26.01 & $<0.001$ \\
\hline Sleep sufficiency imbalance & -0.13 & 0.05 & -0.23 & -0.04 & 695.31 & -2.69 & 0.007 \\
\hline Sex & -0.20 & 0.12 & -0.43 & 0.04 & 118.32 & -1.64 & 0.10 \\
\hline Sleep sufficiency imbalance* sex & 0.14 & 0.07 & -0.01 & 0.27 & 668.44 & 2.07 & 0.039 \\
\hline \multicolumn{8}{|l|}{ Number of awakenings imbalance model } \\
\hline Intercept & 2.61 & 0.11 & 2.40 & 2.82 & 174.85 & 24.64 & $<0.001$ \\
\hline Number of awakenings imbalance & -0.03 & 0.03 & -0.09 & 0.04 & 717.25 & -0.75 & 0.45 \\
\hline Sex & -0.12 & 0.12 & -0.37 & 0.12 & 119.45 & -1.00 & 0.32 \\
\hline Number of awakenings imbalance* sex & 0.05 & 0.05 & -0.05 & 0.14 & 623.23 & 0.99 & 0.32 \\
\hline
\end{tabular}

Notes: Imbalance numbers are undirected, larger numbers mean more imbalance regardless of which parent had more or less. Relationship satisfaction index measured with one question, namely "How satisfied are you with your interaction with your partner today?, "which was scored by each parent every night between $0-4$ with 0 - "not satisfied at all" and 4 - "very satisfied". WASO and TST measured in minutes. Number of wake periods measured in numbers. Waking up first in shared awakenings was measured as the percentage of total shared awakenings. Sleep sufficiency index measured in percentage. Sleep duration measured in minutes. Subjective sleep sufficiency measured in scale $\mathrm{I}-5$.

Abbreviations: Sex, the effect of being female; WASO, wake after sleep onset; TST, total sleep time; SE, standard error; Cl, confidence interval; IQR, interquartile range; $\mathrm{SD}$, standard deviation. 
disturbances than fathers but have a longer sleep duration. Thus, there is little support for the existence of a sleep imbalance when both parents take similar working responsibilities. Future studies may benefit from taking account of who is the primary caregiver during the night, and the impact of different nighttime caregiving strategies on stress and sleep.

\section{Disclosure}

The study was funded by The Swedish Research Council (Vetenskapsrådet), dnr 2018-01902. Göran Härdelin reports grants from Vetenskapsrådet, during the conduct of the study. John Axelsson reports grants from Swedish Research Council, during the conduct of the study; grants from Swedish Research Council, Riksbankens Jubileumsfond, and AFA Insurance, personal fees from the Research Council of Norway, Lifesum, Banqit, Svensk Kiropraktik, and Norra Stockholm Psychiatry, and stocks in Game Intelligence, outside the submitted work. Tina Sundelin reports grants from The Swedish Research Council (Vetenskapsrådet), during the conduct of the study. The authors report no other potential conflicts of interest in this work.

\section{References}

1. Richter D, Kramer MD, Tang NKY, Montgomery-Downs HE, Lemola S. Long-term effects of pregnancy and childbirth on sleep satisfaction and duration of first-time and experienced mothers and fathers. Sleep. 2019;42:4. doi:10.1093/sleep/zsz015

2. Hagen EW, Mirer AG, Palta M, Peppard PE. The sleep-time cost of parenting: sleep duration and sleepiness among employed parents in the Wisconsin Sleep Cohort Study. Am J Epidemiol. 2013;177 (5):394-401. doi:10.1093/aje/kws246

3. Urfer-Maurer N, Weidmann R, Brand S, et al. The association of mothers' and fathers' insomnia symptoms with school-aged children's sleep assessed by parent report and in-home sleepelectroencephalography. Sleep Med. 2017;38:64-70. doi:10.1016/j. sleep.2017.07.010

4. Gay CL, Lee KA, Lee SY. Sleep patterns and fatigue in new mothers and fathers. Biol Res Nurs. 2004;5(4):311-318. doi:10.1177/ 1099800403262142

5. Boergers J, Hart C, Owens JA, Streisand R, Spirito A. Child sleep disorders: associations with parental sleep duration and daytime sleepiness. J Fam Psychol. 2007;21(1):88-94. doi:10.1037/08933200.21.1.88

6. Kalak N, Gerber M, Kirov R, et al. The relation of objective sleep patterns, depressive symptoms, and sleep disturbances in adolescent children and their parents: a sleep-EEG study with 47 families. J Psychiatr Res. 2012;46 (10):1374-1382. doi:10.1016/j.jpsychires.2012.07.006

7. Venn S, Arber S, Meadows R, Hislop J. The fourth shift: exploring the gendered nature of sleep disruption among couples with children. $\mathrm{Br}$ J Sociol. 2008;59(1):79-97. doi:10.1111/j.1468-4446.2007.00183.x

8. Meltzer LJ, Montgomery-Downs HE. Sleep in the family. Pediatr Clin North Am. 2011;58(3):765-774. doi:10.1016/j.pcl.2011.03.010
9. McQuillan ME, Bates JE, Staples AD, Deater-Deckard K. Maternal stress, sleep, and parenting. J Fam Psychol. 2019;33(3):349-359. doi:10.1037/fam0000516

10. Ystrom H, Nilsen W, Hysing M, Sivertsen B, Ystrom E. Sleep problems in preschoolers and maternal depressive symptoms: an evaluation of mother- and child-driven effects. Dev Psychol. 2017;53(12):2261-2272. doi:10.1037/dev0000402

11. da Estrela C, Barker ET, Lantagne S, Gouin JP. Chronic parenting stress and mood reactivity: the role of sleep quality. Stress Health. 2018;34(2):296-305. doi:10.1002/smi.2790

12. Meltzer LJ, Mindell JA. Relationship between child sleep disturbances and maternal sleep, mood, and parenting stress: a pilot study. J Fam Psychol. 2007;21(1):67-73. doi:10.1037/0893-3200.21.1.67

13. Millikovsky-Ayalon M, Atzaba-Poria N, Meiri G. The role of the father in child sleep disturbance: child, parent, and parent-child relationship. Infant Ment Health J. 2015;36(1):114-127. doi:10.1002/imhj.21491

14. Sinai D, Tikotzky L. Infant sleep, parental sleep and parenting stress in families of mothers on maternity leave and in families of working mothers. Infant Behav Dev. 2012;35(2):179-186. doi:10.1016/j. infbeh.2012.01.006

15. Medina AM, Lederhos CL, Lillis TA. Sleep disruption and decline in marital satisfaction across the transition to parenthood. Fam Syst Health. 2009;27(2):153-160. doi:10.1037/a0015762

16. Hasler BP, Troxel WM. Couples' nighttime sleep efficiency and concordance: evidence for bidirectional associations with daytime relationship functioning. Psychosom Med. 2010;72(8):794-801. doi:10.1097/PSY.0b013e3181ecd08a

17. Kouros CD, El-Sheikh M. Within-family relations in objective sleep duration, quality, and schedule. Child Dev. 2016. doi:10.1111/ cdev. 12667

18. Matricciani L, Fraysse F, Grobler AC, Muller J, Wake M, Olds T. Sleep: population epidemiology and concordance in Australian children aged 11-12 years and their parents. BMJ Open. 2019;9 (Suppl3):127-135. doi:10.1136/bmjopen-2017-020895

19. Willoughby K, Julien M, Zendel BR, Curran V. National survey of Canadian residents and program directors regarding parental leave during residency. Can Med Educ J. 2020;11(5):e16-e30. doi:10.36834/cmej.68486

20. Baker FC, Sassoon SA, Kahan T, et al. Perceived poor sleep quality in the absence of polysomnographic sleep disturbance in women with severe premenstrual syndrome. J Sleep Res. 2012;21(5):535-545. doi:10.1111/j.1365-2869.2012.01007.x

21. Berry JO, Jones WH. The parental stress scale: initial psychometric evidence. J Soc Pers Relat. 1995;12(3):463-472. doi:10.1177/ 0265407595123009

22. Gordon AM, Carrillo B, Barnes CM. Sleep and social relationships in healthy populations: a systematic review. Sleep Med Rev. 2021;57:101428. doi:10.1016/j.smrv.2021.101428

23. Peltz JS, Rogge RD, Sturge-Apple ML, O'Connor TG, Pigeon WR. Reciprocal influences among family processes and toddlers' sleep problems. J Fam Psychol. 2016;30(6):720-731. doi:10.1037/ fam0000202

24. Bernier A, Bélanger M-È, Bordeleau S, Carrier J. Mothers, fathers, and toddlers: parental psychosocial functioning as a context for young children's sleep. Dev Psychol. 2013;49(7):1375-1384. doi: $10.1037 / \mathrm{a} 0030024$

25. Ko S-H, Chen C-H, Wang -H-H, Su Y-T. Postpartum women's sleep quality and its predictors in Taiwan. $J$ Nurs Scholarsh. 2014;46 (2):74-81. doi:10.1111/jnu.12053

26. Smallen D, Eller J, Rholes WS, Simpson JA. Perceptions of partner responsiveness across the transition to parenthood. J Fam Psychol. 2021. doi:10.1037/fam0000907

27. Randall AK, Bodenmann G. Stress and its associations with relationship satisfaction. Curr Opin Psychol. 2017;13:96-106. doi:10.1016/j. copsyc. 2016.05 .010 
28. Akerstedt T, Hume K, Minors D, Waterhouse J. Good sleep-its timing and physiological sleep characteristics. J Sleep Res. 1997;6 (4):221-229. doi:10.1111/j.1365-2869.1997.00221.x

29. R Core Team. R: A Language and Environment for Statistical Computing. Vienna AU: R Foundation for Statistical Computing; 2020. Available from: https://www.Rorg/. Accessed October 8, 2021.

30. Bixler EO, Papaliaga MN, Vgontzas AN, et al. Women sleep objectively better than men and the sleep of young women is more resilient to external stressors: effects of age and menopause. J Sleep Res. 2009;18(2):221-228. doi:10.1111/j.1365-2869.2008.00713.x

31. Polo-Kantola P, Laine A, Kronholm E, et al. Gender differences in actual and preferred nocturnal sleep duration among Finnish employed population. Maturitas. 2016;94:77-83. doi:10.1016/j. maturitas.2016.09.002
32. Mallampalli MP, Carter CL. Exploring sex and gender differences in sleep health: a society for women's health research report. J Womens Health. 2014;23(7):553-562. doi:10.1089/jwh.2014.4816

33. Mong JA, Cusmano DM. Sex differences in sleep: impact of biological sex and sex steroids. Philos Trans $R$ Soc Lond B Biol Sci. 2016;371(1688):20150110. doi:10.1098/rstb.2015.0110

34. Van Yperen NW, Buunk BP. A longitudinal study of equity and satisfaction in intimate relationships. Eur J Soc Psychol. 1990;20 (4):287-309. doi:10.1002/ejsp.2420200403

35. WorldEconomicForum. Global gender gap report 2021; 2021. Available from: http://www3.weforum.org/docs/WEF_GGGR_2021. pdf. Accessed October 8, 2021.

\section{Publish your work in this journal}

Nature and Science of Sleep is an international, peer-reviewed, open access journal covering all aspects of sleep science and sleep medicine, including the neurophysiology and functions of sleep, the genetics of sleep, sleep and society, biological rhythms, dreaming, sleep disorders and therapy, and strategies to optimize healthy sleep.
The manuscript management system is completely online and includes a very quick and fair peer-review system, which is all easy to use. Visit http://www.dovepress.com/testimonials.php to read real quotes from published authors. 